\title{
In vivo photoacoustic imaging of chorioretinal oxygen gradients
}

\author{
Ali Hariri \\ Junxin Wang \\ Yeji Kim \\ Anamik Jhunjhunwala \\ Daniel L. Chao \\ Jesse V. Jokerst
}




\title{
In vivo photoacoustic imaging of chorioretinal oxygen gradients
}

\author{
Ali Hariri, ${ }^{a}$ Junxin Wang, ${ }^{a}$ Yeji Kim, ${ }^{b}$ Anamik Jhunjhunwala, ${ }^{c}$ Daniel L. Chao,, and Jesse V. Jokerst ${ }^{a, e, f, *}$ \\ aUniversity of California-San Diego, Nanoengineering Department, La Jolla, California, United States \\ bUniversity of California-San Diego, School of Medicine, La Jolla, California, United States \\ 'University of California-San Diego, Bioengineering Department, La Jolla, California, United States \\ 'University of California-San Diego, Shiley Eye Institute, Department of Ophthalmology, La Jolla, California, United States \\ eUniversity of California-San Diego, Material Science and Engineering Program, La Jolla, California, United States \\ fUniversity of California-San Diego, Radiology Department, La Jolla, California, United States
}

\begin{abstract}
Chorioretinal imaging has a crucial role for the patients with chorioretinal vascular diseases, such as neovascular age-related macular degeneration. Imaging oxygen gradients in the eye could better diagnose and treat ocular diseases. Here, we describe the use of photoacoustic ocular imaging (PAOI) in measuring chorioretinal oxygen saturation ( $\left.\mathrm{CR}-\mathrm{sO}_{2}\right)$ gradients in New Zealand white rabbits $(n=5)$ with ocular ischemia. We observed good correlation $\left(R^{2}=0.98\right)$ between pulse oximetry and PAOI as a function of different oxygen percentages in inhaled air. We then used an established ocular ischemia model in which intraocular pressure is elevated to constrict ocular blood flow, and notice a positive correlation $\left(R^{2}=0.92\right)$ between the injected volume of phosphate buffered saline (PBS) and intraocular pressure (IOP) as well as a negative correlation $\left(R^{2}=0.98\right)$ between $\mathrm{CR}-\mathrm{sO}_{2}$ and injected volume of PBS. The $\mathrm{CR}-\mathrm{sO}_{2}$ was measured before (baseline), during (ischemia), and after the infusion (600- $\mu$ L PBS). The ischemia-reperfusion model did not affect the measurement of the $\mathrm{sO}_{2}$ using a pulse oximeter on the animal's paw, but the chorioretinal PAOI signal showed a nearly sixfold decrease in $\mathrm{CR}-\mathrm{sO}_{2}(n=5, p=0.00001)$. We also observe a sixfold decrease in $\mathrm{CR}-\mathrm{sO}_{2}$ after significant elevation of IOP during ischemia, with an increase close to baseline during reperfusion. These data suggest that PAOI can detect changes in chorioretinal oxygenation and may be useful for application to imaging oxygen gradients in ocular disease. () 2018 Society of Photo-Optical Instrumentation Engineers (SPIE) [DOI: 10.1117/1.JBO.23.3.036005]
\end{abstract}

Keywords: photoacoustic ocular imaging; chorioretina oxygen saturation; oxygen tension; ischemia reperfusion.

Paper 170674RR received Oct. 18, 2017; accepted for publication Feb. 14, 2018; published online Mar. 9, 2018.

\section{Introduction}

The eye is a critical light-sensitive organ ${ }^{1}$ divided into anterior and posterior segments. ${ }^{2}$ The anterior segment includes the cornea, iris, pupil, and ciliary body. The posterior segment includes the vitreous body, retina, choroid, and optic nerve. ${ }^{3}$ The retinal pigment epithelium mostly contains melanin ${ }^{4}$ and plays a major role in energy and oxygen consumption. ${ }^{5}$ Retinal and choroidal circulation delivers oxygen to the inner retinal tissue and photoreceptors, respectively. ${ }^{6}$ Focal retinal hypoxia is thought to underlie the pathophysiology of many ocular diseases, such as diabetic retinopathy, ${ }^{7,8}$ glaucoma, ${ }^{9,10}$ and retinal venous occlusion. ${ }^{11}$ Thus, the ability to image retinal oxygen gradients could be very valuable in treating and diagnosing retinal disease. Also, chorioretinal vasculature imaging has a significant role for diseases, such as neovascular age-related macular degeneration (AMD). ${ }^{12}$

There are four requirements for ocular imaging. First, motion artifacts and image distortion need to be compensated either through high-speed imaging or eye tracking software. Second, imaging should be noninvasive to decrease the risk of side effects. Third, light illumination should be below safety limits to prevent damaging the retina. ${ }^{13}$ Fourth, resolution is an important element of all imaging techniques. However, there are some applications where low-resolution imaging techniques are useful, such as magnetic resonance imaging ${ }^{14}$ and ultrasound. ${ }^{15}$ Current clinical ophthalmology imaging modalities such as optical coherence tomography (OCT), ${ }^{16,17}$ confocal scanning laser ophthalmoscopy, ${ }^{18,19}$ and fundus photography ${ }^{20}$ provide anatomic data on the retina but do not measure oxygen gradients. Hyperspectral imaging ${ }^{21,22}$ has shown potential for imaging oxygen gradients in the eye but still has not been systematically validated, and an artifact of spectral noise from pigmentation, ocular media, etc. can affect hemoglobin oxygen gradients.

More recently, photoacoustic imaging (PAI) has been described as a noninvasive and nonionizing imaging technique that combines optical absorption and ultrasound detection. ${ }^{23-28}$ Photoacoustic signal is generated due to absorption and thermal expansion of endogenous or exogenous molecules using a nanosecond laser pulse followed by detection with a wideband ultrasound transducer. ${ }^{29-31}$ This modality offers high penetration depth due to its weak ultrasound scattering in biological tissue. Oxyhemoglobin and deoxyhemoglobin $\left(\mathrm{HbR}\right.$ and $\left.\mathrm{HbO}_{2}\right)$ as well as melanin are the main optical absorbers in tissue. PAI can measure oxygen saturation $\left(\mathrm{sO}_{2}\right)$ variations based on differences in $\mathrm{HbR}$ and $\mathrm{HbO}_{2}$ absorption. ${ }^{32-35}$ In eye diseases, oxygenation is dysregulated due to increased intraocular pressure (IOP) - the choroidal blood flow decreases as the IOP increases. ${ }^{36}$ In turn, oxygen availability decreases with decreasing $\mathrm{CR}-\mathrm{sO}_{2} \cdot{ }^{36}$ Although PAI is ideally suited to measure oxygen saturation, somewhat surprisingly, most studies in the field have focused only on anatomic imaging. 
Examples of anatomic imaging include de La Zerda et al. ${ }^{37}$ who utilized PAI in swine and rabbit eyes. Their system could image both the anterior and posterior segments of the ocular system. However, $90 \mathrm{~min}$ was required to image a $12-\mathrm{mm} \times 8-\mathrm{mm}$ area. Hu et al. ${ }^{38}$ utilized optical resolution photoacoustic microscopy (OR-PAM) to do in vivo imaging of the anterior segment of a mouse eye; axial and lateral resolution of 15 and $5 \mu \mathrm{m}$ were reported, but $120 \mathrm{~min}$ was required to cover only a 2-mm $\times$ 2-mm area. Liu et al. ${ }^{39}$ and $\mathrm{Wu}$ et al. ${ }^{40}$ improved their OR-PAM system's acquisition time to $20 \mathrm{~min}$ for a $3-\mathrm{mm} \times 3$-mm-image and $6.5 \mathrm{~min}$ for a $2-\mathrm{mm} \times 2$-mm-image, respectively. Jiao et al., ${ }^{41}$ Song et al., ${ }^{42,43}$ and Liu et al. ${ }^{44}$ proposed multimodal ocular imaging using PAI with an optical scanning method and decreased the acquisition time to $2.7 \mathrm{~s}$ for a $2-\mathrm{mm} \times 2-\mathrm{mm}$ image. Recently, Tian et al. ${ }^{45}$ designed chorioretinal imaging of integrated PAM and OCT for rabbits. The acquisition time for a $3-\mathrm{mm} \times 3-\mathrm{mm}$ area was $65 \mathrm{~s}$ with PAM lateral and axial resolution values of 4.1 and $37 \mu \mathrm{m}$, respectively.

Other groups have used microscopy techniques to image oxygen saturation in rodent eyes. Although these techniques offered very high spatial resolution, the penetration depth was limited to a few $\mathrm{mm}$, and the technique required many minutes to map only a few $\mathrm{mm}^{2}$ of tissue. An example of this includes Song et al. $^{43}$ who quantified retinal oxygen saturation in rats. They combined OCT and PAM to measure the retinal oxygenation.

However, to the best of our knowledge, only one study has yet evaluated oxygen saturation in a large animal eye using PAI. In that study, Hennen et al. ${ }^{46}$ used acoustic resolution photoacoustic tomography (AR-PAT) to image the conjunctiva and other areas on the "anterior" of rabbit eyes with 2-Hz temporal resolution. However, a remaining fundamental limitation is oxygen saturation imaging of the "posterior" (chorioretina) of a large animal eye. This has not yet been reported. Indeed, as Hennen and coworkers ${ }^{46}$ stated, such a technique would be useful for evaluating the role of oxidative damage, hypoxia, and ischemia in pathogenesis of various ocular diseases, such as glaucoma, diabetic retinopathy, AMD, and cataract.

Here, we describe high-speed photoacoustic ocular imaging (PAOI) to detect chorioretinal oxygen gradient in an in vivo model of hypoxia as well as an ischemia reperfusion model on albino live rabbits. This PAOI approach is faster and has a simpler optical design than acoustic resolution photoacoustic tomography and PAM techniques. PAOI could monitor $\mathrm{CR}-\mathrm{sO}_{2}$ changes in real time as demonstrated with oxygen tension experiments and ischemia-reperfusion models.

\section{Methods and Materials}

\subsection{Animal Procedures}

New Zealand white rabbits ( 2 to $3 \mathrm{~kg}$ ) were used as animal models. All animal experiments were performed in compliance with the Institutional Animal Care and Use Committee established by University of California San Diego. Ketamine (35 mg/kg) and xylazine $(5 \mathrm{mg} / \mathrm{kg})$ were given via intramuscular injection for anesthesia. The pupils were dilated and anesthetized using $2.5 \%$ phenylephrine hydrochloride, $0.5 \%$ proparacaine hydrochloride, and $1 \%$ tropicamide. The heart rate, peripheral capillary oxygen saturation, respiration rate, and temperature were monitored during the experiment. Animals were placed on a recirculating water blanket kept at $39^{\circ} \mathrm{C}$. An ocular speculum was used to keep the rabbit's eye open and ultrasound gel coupled the eye to the transducer.

\subsection{Photoacoustic Ocular Imaging}

All in vivo imaging was performed using a laser integrated high-frequency ultrasound system (Vevo LAZR, VisualSonics Inc.). A linear array transducer was used with optical fiber bundles integrated to each side (LZ-201, fc $=15 \mathrm{MHz}$, axial resolution $=290 \mu \mathrm{m}$, lateral resolution $=580 \mu \mathrm{m}, \quad B$-mode photoacoustic frame rate $=6 \mathrm{~Hz}, \mathrm{~B}$-mode $\mathrm{CR}-\mathrm{sO}_{2}$ map rate $=0.8 \mathrm{~Hz}$ ). These fiber bundles delivered light from a tunable laser (680 to $970 \mathrm{~nm}$ ). A Q-switched Nd:YAG laser (4- to 6-ns pulse width) with optical parametric oscillator and a repetition rate of $20 \mathrm{~Hz}$ was used to deliver laser energy to the surface of the eye at $8 \pm 0.5 \mathrm{~mJ}$. The transducer can be scanned in one direction to acquire three-dimensional (3-D) data.

\subsection{Oxygen Saturation Measurement Using Photoacoustic Imaging}

The photoacoustic intensity is proportional to the local absorption coefficient $\left(\mu_{a}\right)$, and thus the relative concentration of oxyand deoxyhemoglobin $\left(\mathrm{HbO}_{2}\right.$ and $\left.\mathrm{HbR}\right)$ scan be determined by solving the following equation:

$\mu\left(\lambda_{i}\right)=\varepsilon_{\mathrm{HbR}}\left(\lambda_{i}\right)[\mathrm{HbR}]+\varepsilon_{\mathrm{HbO}_{2}}\left(\lambda_{i}\right)\left[\mathrm{HbO}_{2}\right]$,

where $\varepsilon_{\mathrm{HbR}}\left(\lambda_{i}\right)$ and $\varepsilon_{\mathrm{HbO}_{2}}\left(\lambda_{i}\right)$ are the known molar extinction coefficients $\left(\mathrm{cm}^{-1} \mathrm{M}^{-1}\right)$ of $\mathrm{HbR}$ and $\mathrm{HbO}_{2}$ at wavelength of $\lambda_{i}$, respectively. To calculate $\mathrm{HbO}_{2}$ and $\mathrm{HbR}$ at least two different wavelengths are needed, and $\mathrm{sO}_{2}$ can be obtained as following:

$\mathrm{sO}_{2}(\%)=\frac{\left[\mathrm{HbO}_{2}\right]}{\left[\mathrm{HbO}_{2}\right]+[\mathrm{HbR}]} \times 100$.

In our PAOI, 750 and $850 \mathrm{~nm}$ were used to differentiate the oxy- and deoxyhemoglobin. We used 750 and $850 \mathrm{~nm}$ to differentiate the oxy- and deoxyhemoglobin. These wavelengths have lower light attenuation in tissue and are in the so-called "optical window." 47 This makes them suitable for imaging hemoglobin and deoxyhemoglobin. The 750-nm peak corresponds to deoxyhemoglobin, and the oxyhemoglobin has a peak at $850 \mathrm{~nm}$. Both are in this optical window (600 to $1100 \mathrm{~nm}$ ). Known values for molar extinction coefficients were embedded in the VisualSonics software. ${ }^{48}$

\subsection{Oxygen Saturation Measurement Using Pulse Oximeter}

Pulse oximetry is a noninvasive method to measure the oxygen saturation $\left(\mathrm{sO}_{2}\right)$ of the blood. ${ }^{49} \mathrm{~A}$ commercial peripheral pulse oximeter (CMS60D-VET, Contec Co., UK) was utilized on the rabbit's back paw to validate our oxygen saturation measurement using PAOI.

\subsection{Oxygen Tension Experiment}

To show PAOI's capability to monitor $\mathrm{CR}-\mathrm{sO}_{2}$ and evaluate the response of photoacoustic signal to oxygen tensions, five anesthetized rabbits were administered different percentages of oxygen [5\%,10\%,15\%, 21\% (air), 30\%, and 100\%] with the balance as nitrogen. Pulse oximeter measured $\mathrm{sO}_{2}$ from 
the paw at the same time. We also demonstrated the capability of real-time measurements of $\mathrm{CR}-\mathrm{sO}_{2}$ using PAOI with alternating changes in the level of oxygen from normal air to $100 \%$ oxygen (hyperoxic condition). The results were evaluated with a pulse oximeter. Hyperoxia and normal air were applied to the animal. A maximum intensity projection (MIP) method was utilized to generate 3 -D images.

\subsection{Ischemia Reperfusion Model}

A well-established ischemia-reperfusion model was used to generate chorioretinal intravascular gradients in rabbits $(n=5)$. In this model, a 25-gauge needle was placed in the anterior chamber while the animal was sedated and breathing normal air. A 10-mL syringe containing phosphate buffered saline (PBS) was connected to a syringe pump. PBS then was infused to increase the IOP and induce ischemia. A Tonopen (Reichert Inc.) was used to measure the IOP in this study. To investigate the relationship between IOP, injected PBS volume, and $\mathrm{CR}-\mathrm{sO}_{2}$, we injected 100, 200, 300, and $400 \mu \mathrm{L}$ PBS into the eye. Before and after each injection, the IOP and $\mathrm{CR}-\mathrm{sO}_{2}$ were measured using a Tonopen and PAOI, respectively. The flow rate for this experiment was fixed at $10 \mathrm{~mL} / \mathrm{h}$. Each IOP measurement in this article using Tonopen is an average of three readings.

Next, we confirmed the ability to do real-time imaging using PAOI. Five rabbits were imaged and scanned in this experiment. The flow rate and injection volume were set to $20 \mathrm{~mL} / \mathrm{h}$ and $600 \mu \mathrm{L}$, respectively. A pulse oximeter measured the $\mathrm{sO}_{2}$ from the animal's paw at the same time. The IOP was measured using a Tonopen before ischemia (baseline), during ischemia, and after reperfusion.

\section{Results}

Figure 1 shows the experimental setups in this study. Figure 1(a) shows the oxygen tension experiment, and Fig. 1(b) shows the ischemia-reperfusion setup.

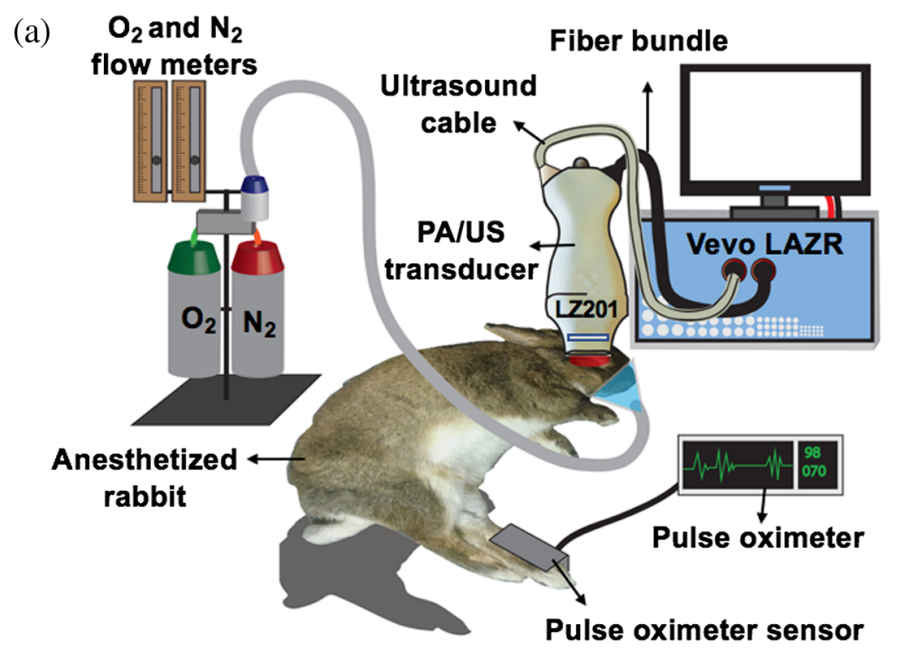

\subsection{Oxygen Tension Experiment}

We first validated whether PAOI could measure $\mathrm{sO}_{2}$ in the eye by comparing $\mathrm{PAOI}-\mathrm{CR}-\mathrm{sO}_{2}$ measurements and pulse oximetry measurements in animals breathing different percentages of oxygen. Figure 2(a) shows the B-mode photoacoustic/ultrasound image of a rabbit eye at $750 \mathrm{~nm}$. The PAOI field of view is limited-only part of the chorioretina (40\% of whole chorioretina) can be imaged using PAOI. This is a result of the light propagation [Fig. 1(b)] and can be improved by tilting the transducer. ${ }^{50}$ The acquisition time for one B-mode $\mathrm{CR}-\mathrm{sO}_{2}$ map is 0.8 frame per second. We see a very strong correlation $\left(R^{2}=98, n=5\right)$ between PAOI and pulse oximetry as reference for $\mathrm{sO}_{2}$ measurement. This result validates the PAOI and oximetry methods.

Also, see in Fig. 3 when one animal transitioning from room air to $100 \% \mathrm{~N}_{2}$ and the accompanying change in $\mathrm{CR}-\mathrm{sO}_{2}$.

Next, we further validated the kinetics of measurement. Figure 4(a) shows synchronization between pulse oximetry and PAOI. The temporal resolution of PAOI was $0.8 \mathrm{~Hz}$, and the refresh rate on the pulse oximeter was $1 \mathrm{~Hz}$. We also validated the reproducibility across $100 \mathrm{~B}$-scans and created 20-mm MIP maps of $\mathrm{CR}-\mathrm{sO}_{2}$ during hyperoxia and normal conditions [Figs. 4(b) and 4(c)]. The coefficient of variation across $100 \mathrm{~B}-$ scans was $1.45 \%$ and $2.15 \%$ for normal and hyperoxia conditions, respectively. The $\mathrm{CR}-\mathrm{sO}_{2}$ values measured with PAOI are significantly lower than the $\mathrm{sO}_{2}$ measurements with a pulse oximeter.

\subsection{Ischemia Reperfusion Model}

We next evaluated the ability of PAOI to detect $\mathrm{CR}-\mathrm{sO}_{2}$ gradients in an ischemia reperfusion model [Fig. 5(a)]. Figures 5(b)-5(e) show the $\mathrm{B}$-mode $\mathrm{CR}-\mathrm{sO}_{2}$ map after injection of $100,200,300$, and $400 \mu \mathrm{L}$ PBS, respectively. We first validated the model by injecting increasing volumes of PBS and noted a proportional increase in IOP as validated with a Tonopen [Fig. 5(f)]. As IOP increases, we see a corresponding decrease in $\mathrm{CR}-\mathrm{sO}_{2}$ levels [Fig. 5(g)]. The injection of $400-\mu \mathrm{L}$ PBS

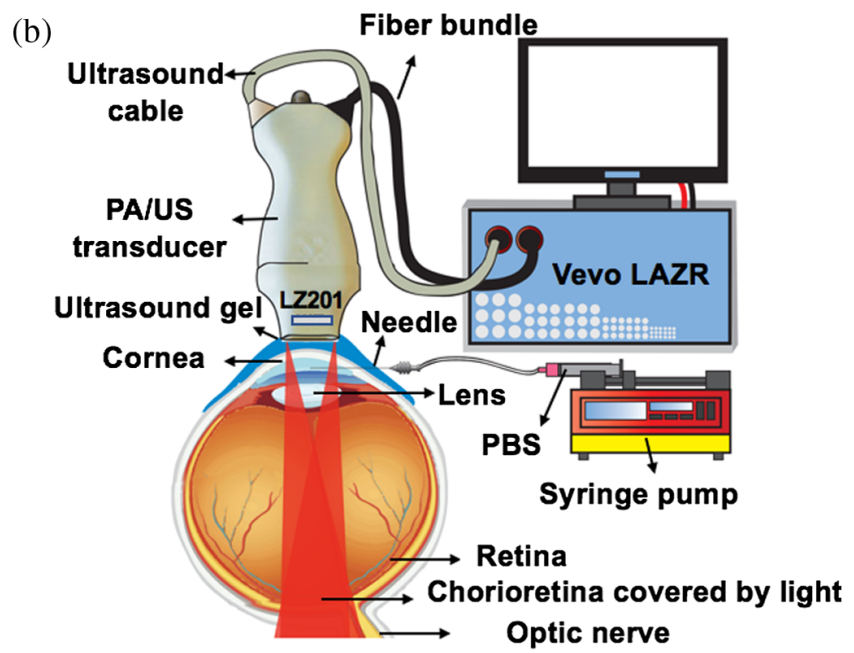

Fig. 1 Illustration of experiment systems. (a) Oxygen tension experiments. Different percentages of oxygen $[5 \%, 10 \%, 15 \%, 21 \%$ (air), 30\%, and $100 \%]$ with the balance as nitrogen were applied to an anesthetized rabbit. A pulse oximeter and $\mathrm{PAOI}$ measured $\mathrm{sO}_{2}$ and $\mathrm{CR}-\mathrm{sO}_{2}$, respectively. (b) Ischemiareperfusion model experiment. A 25-gauge needle was placed in anterior segment of the eye, and a syringe pump controlled the amount of PBS injected into the eye. The direction of light propagation explains the limited field-of-view on the retina surface. 


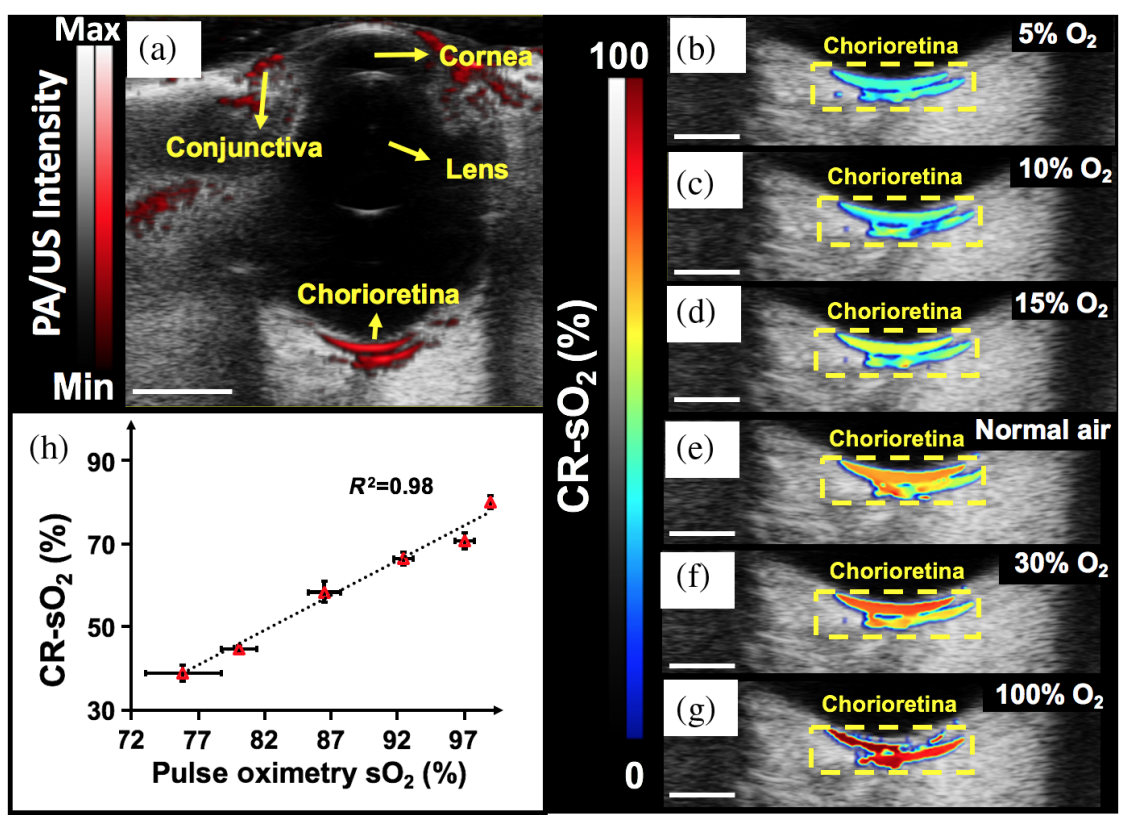

Fig. 2 Evaluation of the PAOI response to oxygen tensions. (a) B-mode photoacoustic/ultrasound image at baseline condition (breathing normal air) including the cornea, conjunctiva, lens, and chorioretina. The image depth is $20 \mathrm{~mm}$. (b) The B-mode $\mathrm{CR}-\mathrm{sO}_{2}$ map when the rabbit is given $5 \% \mathrm{O}_{2}$ and $95 \% \mathrm{~N}_{2}$, (c) $10 \% \mathrm{O}_{2}$ and $90 \% \mathrm{~N}_{2}$, (d) $15 \% \mathrm{O}_{2}$ and $85 \% \mathrm{~N}_{2}$, (e) normal air, (f) $30 \% \mathrm{O}_{2}$ and $70 \% \mathrm{~N}_{2}$, and (g) $100 \%$ $\mathrm{O}_{2}$. For $\mathrm{PAOI}$, the $\mathrm{CR}-\mathrm{sO}_{2}$ measurement frame rate is $0.8 \mathrm{~Hz}$. (h) $\mathrm{PAOl}-\mathrm{CR}-\mathrm{sO}_{2}$ measurements show linear dependence $\left(R^{2}=0.98, n=5\right)$ with pulse oximeter measurements at different oxygen tensions. The error bars show the standard deviation of different five different eyes. All scale bars represent $5 \mathrm{~mm}$.

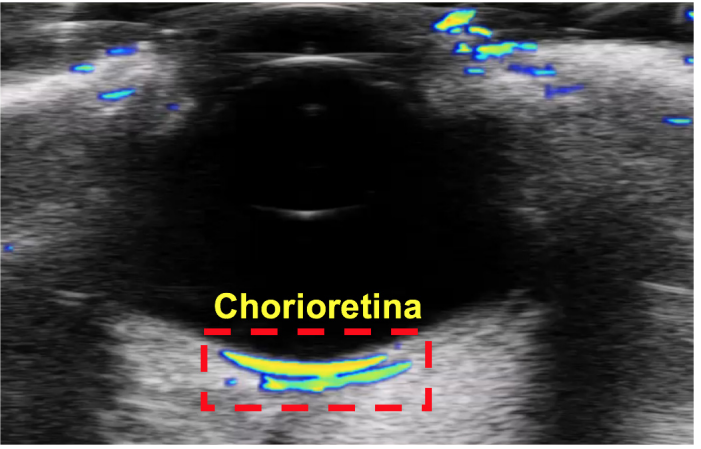

Fig. 3 Still frame from a video showing oxygen tension experiment for one animal transitioning from room air to $100 \% \mathrm{~N}_{2}$ and the accompanying change in $\mathrm{CR}-\mathrm{sO}_{2}$ (Video 1, mp4, $785 \mathrm{~KB}$ [URL: https://doi. org/10.1117/1.JBO.23.3.036005.1]).

resulted in a 4.8-fold increase in IOP and a 1.6-fold decrease in $\mathrm{R}-\mathrm{sO}_{2}$ as measured by Tonopen and PAOI, respectively.

We evaluated whether PAOI could measure changes in chorioretinal oxygenation in an ischemia reperfusion model. IOP was elevated to occlude ocular blood flow using injection of PBS into the anterior chamber. After elevated IOP, we see a steady decrease in $\mathrm{CR}-\mathrm{sO}_{2}$ over $50 \mathrm{~s}$ that reached steady state [Fig. 6(e)]. After decreasing the IOP with subsequent reperfusion, the $\mathrm{CR}-\mathrm{sO}_{2}$ increased - close but not statistically identical to - the baseline values $(n=5, p=0.03)$ [Figs. 6(f) and $6(\mathrm{~g})]$.

Figure 6(a) is a $\mathrm{B}$-mode $\mathrm{CR}-\mathrm{sO}_{2}$ map that was imaged before the start of the ischemia experiment. Figures 6(b) and 6(c) show $\mathrm{CR}-\mathrm{sO}_{2}$ maps 5 and $15 \mathrm{~s}$ after the start of the injection of $600-\mu \mathrm{L}$ PBS in the anterior segment, respectively.

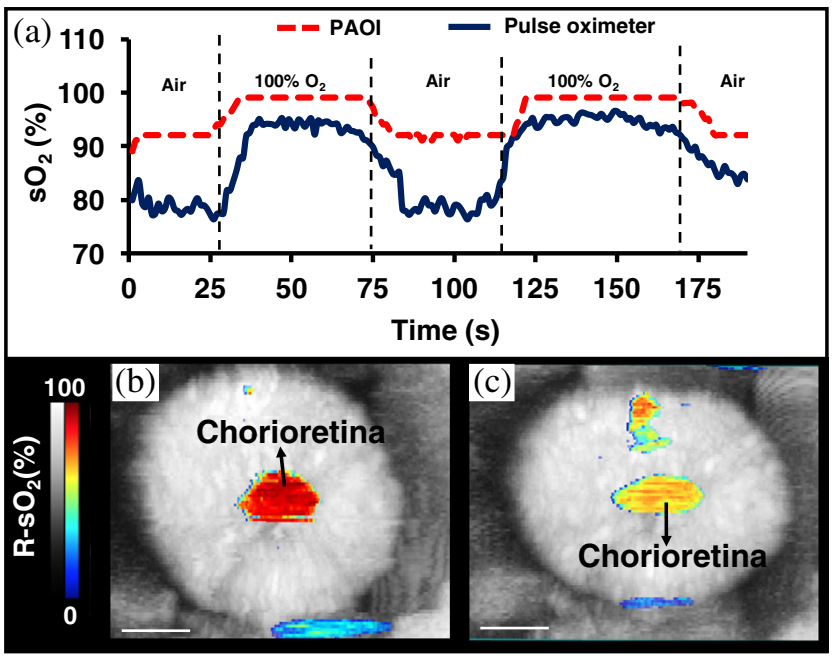

Fig. 4 Validation of real time measurements of $\mathrm{CR}-\mathrm{sO}_{2}$ using $\mathrm{PAOI}$. (a) Real-time monitoring of $\mathrm{sO}_{2}$ using a pulse oximeter and $\mathrm{PAOI}$ in the normal and hyperoxic conditions. PAOI has a frame rate of $0.8 \mathrm{~Hz}$. (b) MIP $\mathrm{CR}-\mathrm{sO}_{2}$ map hyperoxia and (c) MIP $\mathrm{CR}-\mathrm{sO}_{2}$ map during normal breathing condition. The scan size was $20 \mathrm{~mm}$, and the scale bars represent $5 \mathrm{~mm}$.

Figure 6(c) shows an obvious decrease in blood oxygenation during reduced blood flow due to elevated pressure. This pressure was measured with a Tonopen both at baseline and after the injection of $600-\mu \mathrm{L}$ (ischemia). We then removed the needle from the eye and measured the IOP and $\mathrm{CR}-\mathrm{sO}_{2} 15 \mathrm{~min}$ later (reperfusion). Figure 6(d) shows the $\mathrm{B}$-mode $\mathrm{CR}-\mathrm{sO}_{2}$ map in the reperfusion condition. The reperfusion map is not in the exact same plane as the previous condition, because 


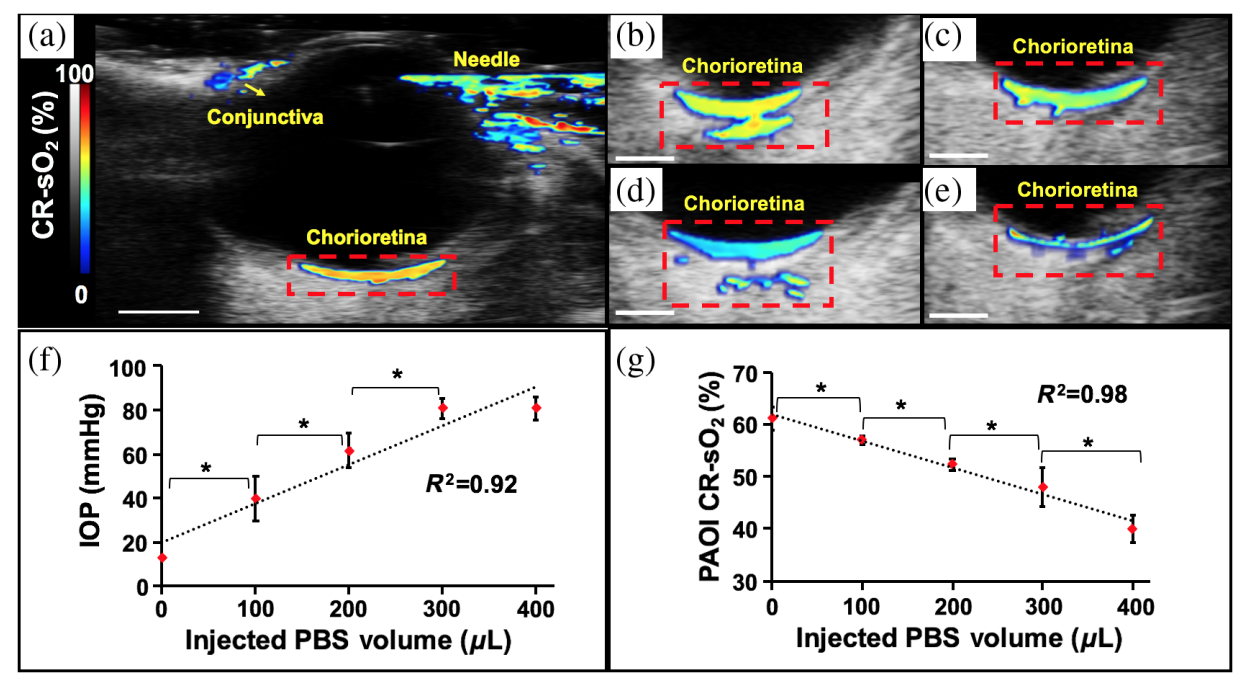

Fig. 5 Investigation of relationship between PBS injected volume, IOP, and $\mathrm{CR}-\mathrm{sO}_{2}$. (a) A b-mode $\mathrm{CR}-\mathrm{sO}_{2}$ map under baseline condition (no PBS injection-no IOP increase). A 25-gauge needle was placed inside the anterior segment of eye. The red dotted rectangle shows the field of view of PAOI on depth of retina. (b) B-mode $\mathrm{CR}-\mathrm{SO}_{2}$ map after injection of $100-\mu \mathrm{L}$ PBS, (c) $200-\mu \mathrm{L}$ PBS, (d) 300- $\mu$ L PBS, and (e) 400- $\mu$ L PBS, (f) linear correlation between IOP and volume of injected PBS in eye $\left(n=5\right.$ eyes; $\left.R^{2}=0.98\right)$. IOP was measured using a Tonopen, and injected PBS volume was controlled by syringe pump. The IOP increased by increasing the PBS volume inside the eye. (g) Correlation between $\mathrm{CR}-\mathrm{sO}_{2}$ and PBS volume inside the eye. Negative correlation is observed between these two parameters $\left(n=5\right.$ eyes; $\left.R^{2}=0.99\right)$. The scale bar is $5 \mathrm{~mm}$. *shows the $p<0.01$. Error bars show a standard deviation among the five different eyes.

we moved the PA/US transducer to measure the IOP and then repositioned it back onto the eye after the IOP measurement.

The PAOI showed a nearly sixfold decrease in $\mathrm{CR}-\mathrm{sO}_{2}$ postinjection of $600 \mu \mathrm{L}(n=5, p=0.00001)$. The $\mathrm{sO}_{2}$ measurement for control areas (conjunctive) confirms that the ischemia only affects the $\mathrm{sO}_{2}$ in the chorioretina and not other parts of the eye. The IOP did not fully return to baseline after reperfusion. It remained elevated relative to baseline $[n=5, p=0.03$; Figs. 6(f) and $6(\mathrm{~g})]$. The $\mathrm{CR}-\mathrm{sO}_{2}$ and IOP are negatively correlated [Figs. 6(f) and $6(\mathrm{~g})]$. The $\mathrm{CR}-\mathrm{sO}_{2}$ decreased with increased IOP $(n=5, p=0.00002)$. Figure 6(e) shows realtime monitoring of both the pulse oximeter and the PAOI during the ischemia-reperfusion experiment. As expected, the ischemia-reperfusion model did not affect $\mathrm{sO}_{2}$ measurements via the pulse oximeter on the animal's paw.

Figure 7 presents the sequence of increase in IOP and subsequent decrease in $\mathrm{CR}-\mathrm{sO}_{2}$.

\section{Discussion}

We demonstrate high speed, simple, and noninvasive PAI to provide spectral, spatial, and temporal data with sufficient resolution to visualize chorioretinal oxygen gradients. This is the first measurement of chorioretina oxygen gradients using an ischemia-reperfusion model and PAI. Rabbits with pigmented eyes (Dutch belted rabbit) were also evaluated using our approach. This strain has pigmented eyes, and we attempted to measure the chorioretinal oxygen gradients using PAOI. The retinal pigmented epithelium is a pigmented layer that is firmly attached to the underlying chorioretinal vessels. This layer contains melanin and absorbs light to generate strong photoacoustic signal. Therefore, we cannot measure the photoacoustic signals from the chorioretina in the pigmented rabbit eye. We used a commercial PAI system (Vevo LAZR, VisualSonics Inc.) and showed that this system is reliable and reproducible for measurement of $\mathrm{CR}-\mathrm{sO}_{2}$ with the frame rate of $0.8 \mathrm{~Hz}$, which is significantly higher than those in the literature. Using this tool, we observed $\mathrm{CR}-\mathrm{sO}_{2}$ gradients both by changing oxygen tension as well as through an ischemia-reperfusion model. Our paper is the first to demonstrate the ability of PAOI to monitor oxygen level on the posterior segment of the eye over such a wide range of oxygen levels. Ketamine-xylazine can reduce oxygen saturation on room air. ${ }^{51,52}$ In addition, we were using the pulse oximeter on the rabbit's paw, which had much more hair than a human finger. This could result in an artificially low oxygenation saturation reading. However, during our initial calibration using oxygen/nitrogen mixtures (Fig. 2), the oxygen saturation reported using PAOI was consistently lower than that reported by the pulse. This difference has been reported in hyperspectral computed tomography system ${ }^{22}$ and AR-PAM ${ }^{46}$ as well. One possible explanation for the difference is that while the peripheral pulse oximeter measures arterial $\mathrm{sO}_{2}$, the PAOI quantifies the $\mathrm{CR}-\mathrm{sO}_{2}$ using the average of both arterial and venous vessels. ${ }^{46}$ Also, the large number of photons are absorbed and attenuated in the anterior segment of eye, such as iris and lens and only limited number of photons is delivered through the eye. Oxygen saturation measurement using PAOI depends on light fluence. The reason of having different numbers from pulse oximeter can be the change of fluence spectrum in depth. ${ }^{53}$ Nevertheless, the PAOI CR $-\mathrm{sO}_{2}$ measurements strongly correlated with systemic measurements of oxygen saturation (pulse oximeter).

One limitation of this approach is balancing spatial resolution and penetration depth. Rabbits' retinal vessels are $20-\mathrm{mm}$ deep. Therefore, we used a $15-\mathrm{MHz}$ transducer with 580 and $290 \mu \mathrm{m}$ as lateral and axial resolution, respectively. Although this could not discriminate individual vessels as in photoacoustics microscopy, ${ }^{43,54}$ we could achieve much deeper penetration. An ideal technique would have good depth penetration and 


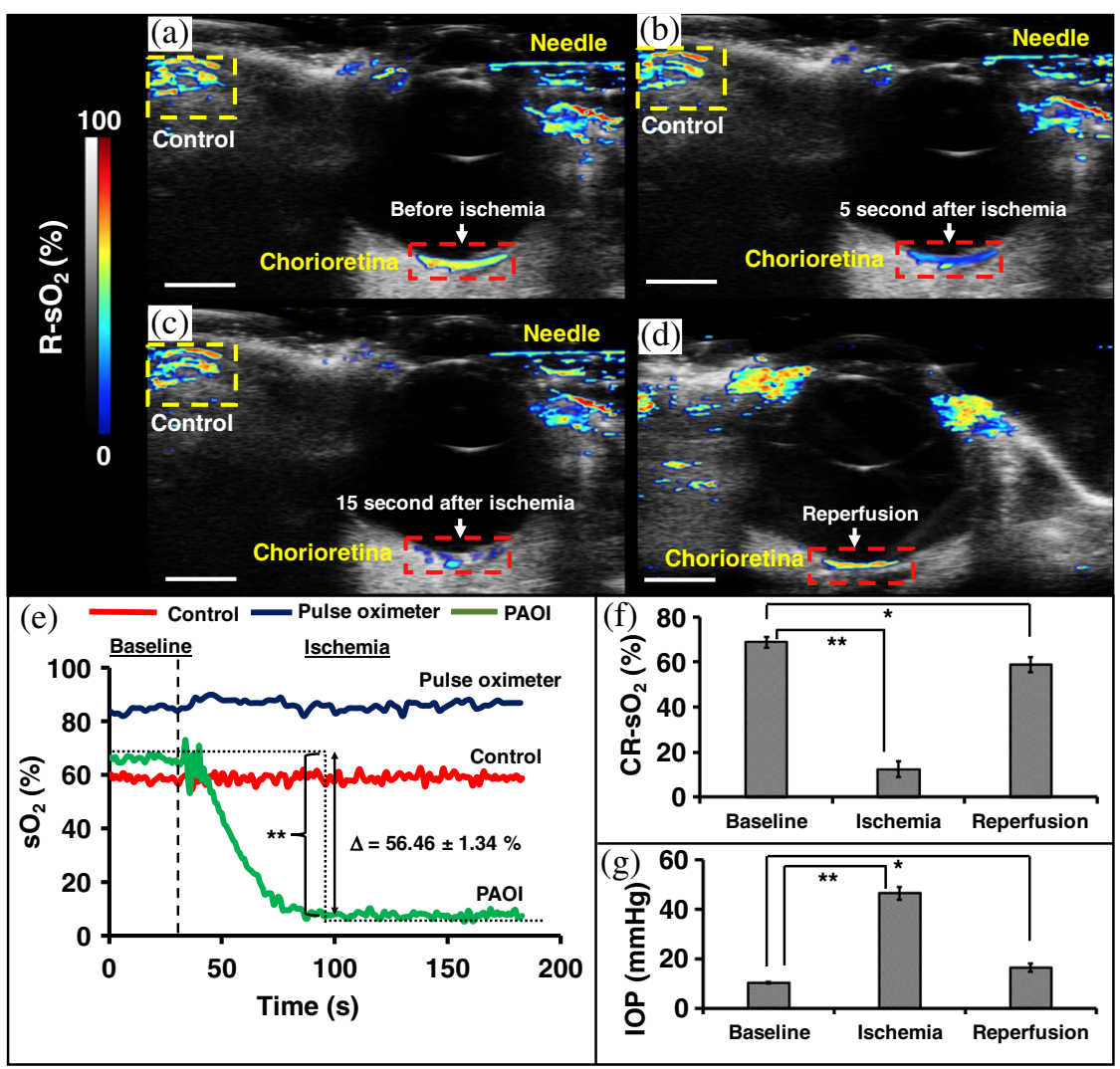

Fig. 6 Real-time evaluation of ischemia-reperfusion model using PAOI. (a) B-mode $\mathrm{CR}-\mathrm{sO}_{2}$ map at baseline condition (no PBS injection-no IOP increase); a 25-gauge needle is inside the anterior segment of eye. Red and yellow dotted rectangles show the retina and control areas, respectively. The $\mathrm{CR}-\mathrm{sO}_{2}$ is $68.76 \pm 2.34$ at baseline (no IOP increase; $10.5 \pm 0.33 \mathrm{~mm} \mathrm{Hg}$ ). (b) The $\mathrm{CR}-\mathrm{sO}_{2}$ map $5 \mathrm{~s}$ after injection of PBS in the eye. Here, the change in the retina map to blue indicates a decrease in $\mathrm{CR}-\mathrm{sO}_{2}$ after $5 \mathrm{~s}$ of IOP. (c) $\mathrm{CR}-\mathrm{sO}_{2}$ gradients after injection of PBS (15 s after start of injection). The IOP increased to $46.5 \pm 2.54 \mathrm{~mm} \mathrm{Hg}$, and the $\mathrm{CR}-\mathrm{sO}_{2}$ decreased to $12.3 \pm 3.54$. (d) $\mathrm{CR}-\mathrm{sO}_{2}$ map in reperfusion condition. The imaging plane is different from other three because we had to remove the transducer and measure the IOP with a Tonopen. $\mathrm{CR}-\mathrm{sO}_{2}$ recovered to $58.87 \pm 3.23$ after the IOP returned to $16.55 \pm 1.67 \mathrm{~mm} \mathrm{Hg}$. (e) Real-time monitoring of $\mathrm{CR}-\mathrm{sO}_{2}$ and $\mathrm{sO}_{2}$ using PAOI and pulse oximetry. The $\Delta$ shows $\mathrm{sO}_{2}$ difference between baseline and ischemia. (f) Statistical analysis of $\mathrm{CR}-\mathrm{sO}_{2}$ for five eyes under different conditions including baseline, ischemia, and reperfusion using PAOI. The $\mathrm{PAOI}$ shows sixfold decrease in $\mathrm{CR}-\mathrm{sO}_{2}$ during ischemia $(n=5)$. There was a very significant change between baseline and ischemia and a significant different in $\mathrm{CR}-\mathrm{SO}_{2}$ from baseline to reperfusion. (g) Statistical analysis of IOP for five eyes at baseline, ischemia, and reperfusion using Tonopen. There is negative correlation between IOP and $\mathrm{CR}-\mathrm{sO}_{2}$. Error bars shows the standard deviation of five different eyes. Here, ${ }^{*}$ indicates $p<0.05$ and ${ }^{* *}$ indicates $p<0.0001$.

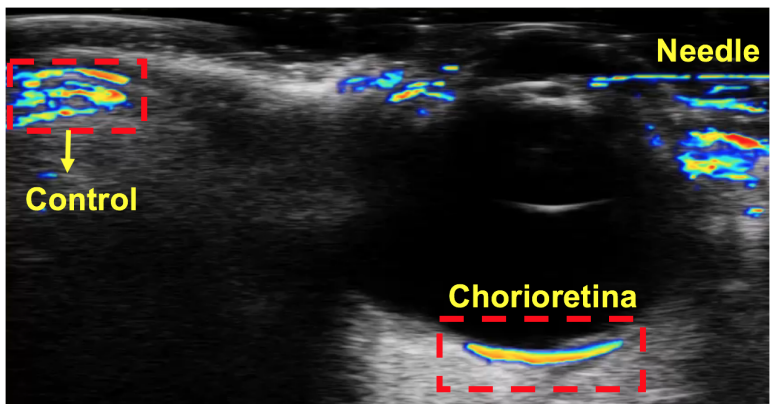

Fig. 7 Still frame from a video showing real-time evaluation of ischemia-reperfusion model using PAOI (Video 2, mp4, $1 \mathrm{MB}$ [URL: https:// doi.org/10.1117/1.JBO.23.3.036005.2]). 50-micron resolution to determine if specific sections of the retina are hypoxic. This current PAOI approach cannot discriminate between the retina and the choroid, and the area of study includes both features.

Previous work ${ }^{22}$ applied ischemia-reperfusion model on a rabbit's eye and measured the $\mathrm{CR}-\mathrm{sO}_{2}$ using hyperspectral computed tomography system. This work used an elevated reservoir of balanced salt solution (BSS) to increase the IOP, but there was no precise control on the amount of injected BSS and IOP in the eye. Here, we used a syringe pump to more precisely control the injected volume (Fig. 5). With this model, the CR $\mathrm{sO}_{2}$ decreased with increasing volume of injected PBS due to interrupted blood flow. In $\mathrm{CR}-\mathrm{sO}_{2}$ map, we found that $10 \mathrm{~s}$ after starting the PBS injection, blood flow to the chorioretinal rapidly dropped (Fig. 6). The $\mathrm{CR}-\mathrm{sO}_{2}$ during reperfusion was lower than baseline $(n=5, p=0.03)$ likely because of compensatory mechanisms of repair in the eye. 
Another limitation is the use of a Tonopen designed for human on rabbit subjects. Mermoud et al. ${ }^{55}$ demonstrated that the Tonopen significantly underestimated the pressure from 5 to $80 \mathrm{mmHg}$. However, they showed high correlation between the transducer pressure and Tonopen as well. This means that the IOP numbers reported with the Tonopen on rabbits might be lower than the true IOP. The IOP numbers are likely 10 to $25 \mathrm{mmHg}$, because the Tonopen was used after the needle was removed from the eye after injection. Nevertheless, the good correlation seen here between injected volume of saline, Tonopen, and PAOI suggests that this model is indeed creating ischemia. Figure 6 shows changes in IOP (from baseline to $46.5 \pm 2.54 \mathrm{~mm} \mathrm{Hg}$ ) with just one step injection of $600 \mu \mathrm{L}$ PBS in the eye.

The maximum permissible exposure (MPE) from the American National Standard Institute ${ }^{56}$ for 700 - to $1050-\mathrm{nm}$ laser light with 10 -s illumination is $5.0 \mathrm{C}_{\mathrm{A}} \mathrm{C}_{\mathrm{E}} \times 10^{-7} \mathrm{~J} / \mathrm{cm}^{2}$. Here, there is a $20-\mathrm{Hz}$ repetition rate and a 5 -ns pulse width. This results in $\sim 1 \mu \mathrm{s}$ of actual laser pulse illumination time. With 700- to 1050-nm laser light and based on illumination specifications, $\quad C_{A}=10^{2(\lambda-0.700)}$ and $C_{E}=\alpha /\left(\alpha_{\min } \alpha_{\max }\right)$. Here, $\lambda$ and $\alpha$ are the radiation wavelength and the full angle retina exposure, respectively. ${ }^{57}$ For $\lambda=750 \mathrm{~nm}$ and $\alpha=$ 1100 miliradians, the MPE is $\sim 5.1 \mathrm{~mJ} / \mathrm{cm}^{2}$. Our approach used $\sim 4.5 \mathrm{~mJ} / \mathrm{cm}^{2}$. Hennen et al. used $5 \mathrm{~mJ} / \mathrm{cm}^{2}$ and showed no damage via histology one after imaging session. To decrease this risk, light-emitting diodes (LED) with much lower incident fluence could also be used. ${ }^{58,59}$ We already characterized the LED-based PAI system in terms of penetration depth. Temporal resolution up to $30 \mathrm{~Hz}$ and signal-to-noise ratio of higher than 3 can be achieved for embedded pencil lead inside the chicken breast. We also evaluated application of LED system for monitoring chorioretina vessels on ex vivo rabbit eyes with frame rate of $15 \mathrm{~Hz}^{59}$

\section{Conclusion}

In this paper, we describe a high-speed PAOI technique to monitor chorioretina oxygen gradients in vivo. This the first report of PAI applied to $\mathrm{CR}-\mathrm{sO}_{2}$ measurements of ischemia-reperfusion in a large animal eye. To the best of our knowledge, a frame rate of $0.8 \mathrm{~Hz}$ for measuring $\mathrm{CR}-\mathrm{sO}_{2}$ is the highest frame rate yet reported, allowing for dynamic measurements of chorioretinal oxygenation in real time. We demonstrate a very strong correlation between $\mathrm{PAOI} \mathrm{CR}-\mathrm{sO}_{2}$ and pulse oximetry with varying levels of inspired oxygen, and we demonstrate a dramatic decrease in oxygen gradients during ocular ischemia. These experiments demonstrate that PAOI can serve as a dynamic measurement oxygen gradients in the eye and may be useful in the future for applications in ocular disease.

\section{Disclosures}

The authors have no relevant financial interests in this article and no potential conflicts of interest to disclose.

\section{Acknowledgments}

Jesse V. Jokerst acknowledges funding from NIH HL117048 and HL137187 and infrastructure from S10 OD021821. The authors also thank the American Cancer Society Institutional Research (Grant No. 14-250-42) provided through the Moores Cancer Center, University of California, San Diego. We also knowledge UC San Diego for funding via the Frontiers of Innovation Scholars Program (FISP \#3-G3005). The authors acknowledge Kristyn Huffman from Dr. Cheng's Lab at UC San Diego for her assistance with measuring IOP in this study.

\section{References}

1. J. I. Korenbrot, "Speed, sensitivity, and stability of the light response in rod and cone photoreceptors: facts and models," Prog. Retinal Eye Res. 31(5), 442-466 (2012).

2. C. E. Willoughby et al., "Anatomy and physiology of the human eye: effects of mucopolysaccharidoses disease on structure and functiona review," Clin. Exp. Ophthalmol. 38(s1), 2-11 (2010).

3. P. Artal, A. Benito, and J. Tabernero, "The human eye is an example of robust optical design," J. Vision 6(1), 1 (2006).

4. B. Winn et al., "Factors affecting light-adapted pupil size in normal human subjects," Invest. Ophthalmol. Visual Sci. 35(3), 1132-1137 (1994).

5. D.-Y. Yu and S. J. Cringle, "Oxygen distribution and consumption within the retina in vascularised and avascular retinas and in animal models of retinal disease," Prog. Retinal Eye Res. 20(2), 175-208 (2001).

6. C. Delaey and J. Van de Voorde, "Regulatory mechanisms in the retinal and choroidal circulation," Ophthalmic Res. 32(6), 249-256 (2000).

7. B. Khoobehi et al., "Retinal arterial and venous oxygen saturation is altered in diabetic patientsretinal oxygen saturation trend in diabetes," Invest. Ophthalmol. Visual Sci. 54(10), 7103-7106 (2013).

8. S. H. Hardarson and E. Stefánsson, "Retinal oxygen saturation is altered in diabetic retinopathy," Br. J. Ophthalmol. 96(4), 560-563 (2012).

9. S. H. Hardarson et al., "Glaucoma filtration surgery and retinal oxygen saturation," Invest. Ophthalmol. Visual Sci. 50(11), 5247-5250 (2009).

10. M. Ito et al., "Oxygen saturation levels in the juxta-papillary retina in eyes with glaucoma," Exp. Eye Res. 86(3), 512-518 (2008).

11. T. S. Eliasdottir et al., "Retinal oximetry measures systemic hypoxia in central nervous system vessels in chronic obstructive pulmonary disease," PLoS One 12(3), e0174026 (2017).

12. P. Keane and S. Sadda, "Imaging chorioretinal vascular disease," Eye 24(3), 422-427 (2010).

13. W. Liu and H. F. Zhang, "Photoacoustic imaging of the eye: a mini review," Photoacoustics 4(3), 112-123 (2016).

14. G. Zuccoli et al., "Susceptibility weighted imaging depicts retinal hemorrhages in abusive head trauma," Neuroradiology 55(7), 889-893 (2013).

15. M. Restori, "Imaging the vitreous: optical coherence tomography and ultrasound imaging," Eye 22(10), 1251-1256 (2008).

16. J. Yi et al., "Visible-light optical coherence tomography for retinal oximetry," Opt. Lett. 38(11), 1796-1798 (2013).

17. T. E. De Carlo et al., "A review of optical coherence tomography angiography (OCTA)," Int. J. Retina Vitreous 1(1), 5 (2015).

18. P. Sharp et al., "The scanning laser ophthalmoscope-a review of its role in bioscience and medicine," Phys. Med. Biol. 49(7), 1085-1096 (2004).

19. B. I. Gramatikov, "Modern technologies for retinal scanning and imaging: an introduction for the biomedical engineer," Biomed. Eng. Online 13(1), 52 (2014).

20. R. Bernardes, P. Serranho, and C. Lobo, "Digital ocular fundus imaging: a review," Ophthalmologica 226(4), 161-181 (2011).

21. V. Nourrit et al., "High-resolution hyperspectral imaging of the retina with a modified fundus camera," J. Fr. D'ophtalmol. 33(10), 686-692 (2010).

22. A. H. Kashani et al., "Hyperspectral computed tomographic imaging spectroscopy of vascular oxygen gradients in the rabbit retina in vivo," PLoS One 6(9), e24482 (2011).

23. M. Xu and L. V. Wang, "Photoacoustic imaging in biomedicine," Rev. Sci. Instrum. 77(4), 041101 (2006).

24. A. Hariri et al., "Development of low-cost photoacoustic imaging systems using very low-energy pulsed laser diodes," J. Biomed. Opt. 22(7), 075001 (2017).

25. J. Wang et al., "A nanoscale tool for photoacoustic-based measurements of clotting time and therapeutic drug monitoring of heparin," Nano Lett. 16(10), 6265-6271 (2016).

26. J. E. Lemaster and J. V. Jokerst, "What is new in nanoparticle-based photoacoustic imaging?" Wiley Interdiscip. Rev. Nanomed. Nanobiotechnol. 9(1), e1404 (2017). 
27. M. Mehrmohammadi et al., "Photoacoustic imaging for cancer detection and staging," Curr. Mol. Imaging 2(1), 89-105 (2013).

28. Y. Jiang and K. Pu, "Advanced photoacoustic imaging applications of near-infrared absorbing organic nanoparticles," Small 13, 1700710 (2017).

29. L. V. Wang and S. Hu, "Photoacoustic tomography: in vivo imaging from organelles to organs," Science 335(6075), 1458-1462 (2012).

30. R. H. Silverman et al., "High-resolution photoacoustic imaging of ocular tissues," Ultrasound Med. Biol. 36(5), 733-742 (2010).

31. F. Kong et al., "High-resolution photoacoustic imaging with focused laser and ultrasonic beams," Appl. Phys. Lett. 94(3), 033902 (2009).

32. H. F. Zhang et al., "Imaging of hemoglobin oxygen saturation variations in single vessels in vivo using photoacoustic microscopy," Appl. Phys. Lett. 90(5), 053901 (2007).

33. H. F. Zhang et al., "Functional photoacoustic microscopy for highresolution and noninvasive in vivo imaging," Nat. Biotechnol. 24(7), 848-851 (2006).

34. C. J. Arthuis et al., "Real-time monitoring of placental oxygenation during maternal hypoxia and hyperoxygenation using photoacoustic imaging," PLoS One 12(1), e0169850 (2017).

35. T. Mitcham et al., "Photoacoustic-based $\mathrm{SO}_{2}$ estimation through excised bovine prostate tissue with interstitial light delivery," Photoacoustics 7, 47-56 (2017)

36. W. Abdallah et al., "Vitreal oxygenation in retinal ischemia reperfusion," Invest. Ophthalmol. Visual Sci. 52(2), 1035-1042 (2011).

37. A. de La Zerda et al., "Photoacoustic ocular imaging," Opt. Lett. 35(3), 270-272 (2010).

38. S. Hu et al., "Label-free photoacoustic ophthalmic angiography," Opt. Lett. 35(1), 1-3 (2010).

39. W. Liu et al., "In vivo corneal neovascularization imaging by opticalresolution photoacoustic microscopy," Photoacoustics 2(2), 81-86 (2014).

40. N. Wu et al., "High-resolution dual-modality photoacoustic ocular imaging," Opt. Lett. 39(8), 2451-2454 (2014).

41. S. Jiao et al., "Photoacoustic ophthalmoscopy for in vivo retinal imaging," Opt. Express 18(4), 3967-3972 (2010).

42. W. Song et al., "Integrating photoacoustic ophthalmoscopy with scanning laser ophthalmoscopy, optical coherence tomography, and fluorescein angiography for a multimodal retinal imaging platform," J. Biomed. Opt. 17(6), 061206 (2012).

43. W. Song et al., "A combined method to quantify the retinal metabolic rate of oxygen using photoacoustic ophthalmoscopy and optical coherence tomography," Sci. Rep. 4, 6525 (2014).

44. T. Liu et al., "Fundus camera guided photoacoustic ophthalmoscopy," Curr. Eye Res. 38(12), 1229-1234 (2013).

45. C. Tian et al., "Noninvasive chorioretinal imaging in living rabbits using integrated photoacoustic microscopy and optical coherence tomography," Opt. Express 25(14), 15947-15955 (2017).

46. S. N. Hennen et al., "Photoacoustic tomography imaging and estimation of oxygen saturation of hemoglobin in ocular tissue of rabbits," Exp. Eye Res. 138, 153-158 (2015).

47. N. Won et al., "Imaging depths of near-infrared quantum dots in first and second optical windows," Mol. Imaging 11(4), 338-352 (2012).

48. S. L. Jacques and S. Prahl, "Absorption spectra for biological tissues," Oregon Graduate Institute, http://omlc.ogi.edu/classroom/ece532/ class $3 /$ muaspectra.html (2004).

49. S. J. Barker, K. K. Tremper, and J. Hyatt, "Effects of methemoglobinemia on pulse oximetry and mixed venous oximetry," Anesthesiology 70(1), 112-117 (1989).

50. S. Y. Nam and S. Y. Emelianov, "Array-based real-time ultrasound and photoacoustic ocular imaging," J. Opt. Soc. Korea 18(2), 151-155 (2014).

51. K. J. Liu et al., "Assessment of cerebral $\mathrm{pO}_{2}$ by EPR oximetry in rodents: effects of anesthesia, ischemia, and breathing gas," Brain Res. 685(1), 91-98 (1995).
52. K. J. Liu et al., "Effect of anesthesia on cerebral tissue oxygen and cardiopulmonary parameters in rats," in Oxygen Transport to Tissue XVIII, E. M. Nemoto et al., Eds., pp. 33-39, Springer, Boston, Massachusetts (1997).

53. C. L. Bayer et al., "Ultrasound-guided spectral photoacoustic imaging of hemoglobin oxygenation during development," Biomed. Opt. Express 8(2), 757-763 (2017).

54. W. Song et al., "In vivo photoacoustic chorioretinal vascular imaging in albino mouse," Chin. Opt. Lett. 12(5), 051704 (2014).

55. A. Mermoud et al., "Measurement of rabbit intraocular pressure with the tono-pen," Ophthalmologica 209(5), 275-277 (1995).

56. ANSI, "American national standard for the safe use of lasers," Z136.1, American National Standards Institute, Inc., New York (1993).

57. F. C. Delori, R. H. Webb, and D. H. Sliney, "Maximum permissible exposures for ocular safety (ANSI 2000), with emphasis on ophthalmic devices," J. Opt. Soc. Am. A 24(5), 1250-1265 (2007).

58. T. Agano et al., "Attempts to increase penetration of photoacoustic system using LED array light source," Proc. SPIE 9323, $93233 Z$ (2015).

59. A. Hariri et al., "The characterization of an economic and portable LEDbased photoacoustic imaging system to facilitate molecular imaging," Photoacoustics 9, 10-20 (2018).

Ali Hariri is a PhD student in the nanoengineering department at the University of California-San Diego. He received his master's and bachelor's degrees in biomedical engineering from Sharif University of Technology and Amirkabir University of Technology in Iran. He worked on developing different configurations of photoacoustic imaging technique including computed tomography and microscopy (both acoustic and optical resolution).

Junxin Wang is a PhD student in the Nanoengineering Department at UC San Diego. He received his MS degree in electro-optics from the University of Dayton and his BS degree in optical information science and technology from Changchun University of Science and Technology. Currently, he is working on biomolecular imaging using nanoparticles and extracellular vesicles.

Yeji Kim received her bachelor of arts degree in human biology from Stanford University. Currently, she is pursuing her MD degree at the University of California San Diego School of Medicine.

Anamik Jhunjhunwala received his BS degree in bioengineering, biotechnology and his BA degree in economics from the University of California San Diego. As an undergraduate, he studied organon-a-chip systems for characterization and diagnostic purposes, the interaction of methylene blue with heparin and SDS, and copper sulfide nanoparticles for the imaging of ovarian cancer. He also worked on the development of a molecular diagnostic device to identify different bacterial strains using digital high resolution melt analysis.

Daniel L. Chao is an assistant professor of ophthalmology at the Shiley Eye Institute. He received his BS degree in biomedical engineering from Virginia Commonwealth University and his MD and $\mathrm{PhD}$ degrees in neurosciences from Stanford University. His clinical focus is on the medical and surgical treatment of retinal diseases, with a special interest in macular degeneration and diabetic retinopathy. $\mathrm{He}$ is involved in multiple interdisciplinary collaborations to develop imaging modalities and therapeutic approaches for retinal diseases.

Jesse V. Jokerst received his BS degree cum laude at Truman State University. After a PhD in chemistry at UT Austin with John McDevitt, he completed his postdoc with Sam Gambhir at Stanford Radiology. Now an assistant professor in the Department of Nanoengineering at UC San Diego, the Jokerst group is eager to collaborate on projects broadly related to human health and nanotechnology. 